\title{
ESTRATEGIAS IDENTITARIAS DE LOS EMIGRANTES CANARIOS RETORNADOS DE VENEZUELA ${ }^{1}$ \\ IDENTITY STRATEGIES OF CANARY EMIGRANTS RETURNED FROM VENEZUELA
}

DANIEL BURASCHI

Resumen: Los emigrantes canarios retornados de Venezuela son sujetos que por su trayectoria migratoria se encuentran en un horizonte cultural en el cual confluyen elementos propios de tres culturas: la cultura canaria que habían abandonado, elementos del horizonte cultural venezolano en donde han vivido y elementos de la sociedad canaria actual a la cual regresan. En este artículo se presentan los resultados de una investigación sobre las estrategias identitarias llevadas a cabo por los emigrantes retornados de Venezuela residentes en Tenerife con el objetivo de generar una tipología sistemática de las mismas y estudiar las interacciones a través de las cuales estos emigrantes construyen y reconstruyen sus identidades culturales a lo largo de su trayectoria migratoria.

En este análisis se ha seguido el esquema metodológico propio de la teoría fundamentada, es decir, una estrategia inductiva de análisis cualitativo. El resultado final de esta investigación ha sido la identificación y categorización de dos procesos sociales básicos relacionados con las dinámicas identitarias: las estrategias pragmáticas y las estrategias ontológicas.

\section{Universidad de Castilla-La Mancha.}

1 Esta investigación se ha desarrollado en el marco de las investigaciones del Grupo de Estudios sobre Migraciones, Interculturalidad y Ciudadanía (GIEMIC), de la Universidad de Castilla-La Mancha. 
Palabras clave: Estrategias identitarias, emigración canaria a Venezuela, emigrantes retornados.

Abstract: Canary emigrants returned from Venezuela are individuals whose migration experience places them in a cultural space in which converge typical elements from three cultural horizons: the Canary culture they abandoned, the Venezuelan culture they lived in, and the current Canary culture they are returning to.

This article describes the results of a research on the identity strategies developed by Canary emigrants returned from Venezuela and currently residing in Tenerife. The objective is the creation of a systematic typology of these strategies and the analysis of the interactions through which these emigrants construct and reconstruct their cultural identities throughout their migrant course.

The methodological schema followed in this research has been Grounded Theory, an inductive strategy of qualitative analysis. The final result of this research is the identification and categorisation of two basic social processes related to the identity dynamics: the pragmatic strategies and the ontological strategies.

Keywords: identity strategies, Canary emigration to Venezuela, returned emigrants.

Las migraciones son experiencias vitales intensas que conllevan una reconstrucción profunda de la identidad de un sujeto. Una persona migrante que se encuentra en un nuevo país experimenta un cambio de su contexto social y cultural que puede poner en crisis su sentimiento de pertenencia y su identidad.

Los emigrantes retornados se reencuentran en un espacio cultural que habían abandonado durante décadas, teniendo que gestionar tres horizontes culturales distintos: la sociedad desde la cual emigraron, el país en el cual residieron y la sociedad que les vuelve a acoger y que resulta, inevitablemente, no ser la misma que habían dejado. El retorno, en particular en los casos de emigrantes que han pasado décadas en el extranjero, constituye una importante ruptura de la vida cotidiana que implica un difícil proceso de redefinición y reestructuración identitaria. Los emigrantes que retornan a Canarias vuelven con un repertorio identitario y un horizonte cultural que a menudo no coincide con el de la sociedad canaria actual. 
Los emigrantes retornados redefinen y reestructuran sus identidades a través de estrategias identitarias y de procesos que implican una negociación, no solamente entre elementos de la cultura canaria y de la cultura del país donde habían emigrado, sino también una gestión de las contradicciones entre lo que ellos consideraban propio de su horizonte cultural canario y las características de la sociedad canaria actual. Lo interesante de estos emigrantes es que se identifican con la sociedad a la que regresan al mismo tiempo que la desconocen.

El estudio de estas estrategias nos permite comprender los complejos procesos de reintegración de estos emigrantes en la sociedad canaria, así como las dinámicas de construcción y reconstrucción identitaria.

\section{VENEZUELA, LA OCTAVA ISLA}

Entre Canarias y Venezuela existen fuertes lazos históricos y culturales derivados de una larga y consolidada tradición migratoria.

La emigración a tierras americanas es un hecho consustancial al Archipiélago desde el mismo momento del descubrimiento del Nuevo Mundo (Hernández González, 2005: 9).

Los dos países representan polos de un amplio campo migratorio caracterizado históricamente por los flujos hacia Venezuela y, en las últimas décadas, por importantes flujos hacia Canarias constituidos por inmigrantes venezolanos y emigrantes españoles retornados. Como subraya Godenau (2011:79) el retorno de los canarios está determinado por las características del flujo de emigración a Venezuela, el porvenir económico y social que han vivido los emigrantes, la percepción de la evolución de las condiciones de vida en la sociedad canaria y las condiciones de la vuelta. En este sentido, el retorno es una expresión específica de las redes migratorias que unen Venezuela y Canarias.

Venezuela ha sido uno de los destinos privilegiados de la emigración canaria, y su estrecha relación con el archipiélago se puede resumir con un código en vivo particularmente recurrente: «Venezuela, la octava isla canaria». 
No hay canario que no tenga un familiar o un conocido que no haya emigrado a la octava isla (Mora, 2011:125).

Si la emigración canaria a América ha sido objeto de un importante corpus de estudios históricos y sociológicos (Ascanio, 2001), no pasa lo mismo con la emigración de retorno ${ }^{2}$ que, salvo algunas excepciones $^{3}$ :

Apenas se ha evaluado y explicado [...] como un fenómeno social, económico y espacial de gran trascendencia e impacto, en tanto que corresponde a la fase centrípeta de las migraciones exteriores (Delgado y Ascanio, 1998:12).

\section{LAS ESTRATEGIAS IDENTITARIAS}

El objeto de esta investigación es el análisis de las estrategias identitarias de los emigrantes canarios retornados de Venezuela, con la finalidad de generar una tipología sistemática de las mismas y estudiar las interacciones a través de las cuales los emigrantes retornados construyen y reconstruyen sus identidades culturales a lo largo de su trayectoria migratoria. Al mismo tiempo se aspira a abrir camino en un área de estudio poco explorada y de gran importancia para comprender los procesos de reintegración socio-culturales de los emigrantes retornados.

Los emigrantes canarios retornados de Venezuela son sujetos que, por su trayectoria migratoria, se encuentran en un espacio social y cultural en el cual confluyen elementos propios de la cultura canaria que habían abandonado, elementos del horizonte cultural venezolano y elementos de la sociedad canaria actual. Las estrategias identitarias son procesos puestos en marcha por las personas migrantes para hacer frente a los diferentes elementos, a veces incompatibles, que conforman sus diferentes horizontes culturales.

2 Según Carnero y Nuez (2011) desde los años Ochenta han vuelto a Canarias unas 14.600 personas.

3 Entre los cuales hay que destacar Colectivo 78 (1981), Martín (1982), Rivero (1987), Delgado y Ascanio (1998), Ascanio (2000 y 2002) Carnero y Nuez (2011). 
En este estudio entendemos la identidad cultural no como una esencia, sino como un proceso continuo y dinámico de identificaciones ${ }^{4}$. La identidad es el producto de una negociación entre actores sociales y no se mantiene excluyendo el cambio, sino negociando e integrando los cambios en una continuidad de sentido. El constructo de estrategia identitaria ha sido desarrollado sobre todo por Camilleri (1990) quién inaugurará, a partir de los años ochenta, una fecunda línea de estudios centrada, fundamentalmente, en las dinámicas identitarias de los inmigrantes magrebíes en Francia. Según Camilleri, las estrategias identitarias permiten al sujeto defender su existencia y su visibilidad social, su integración en la comunidad al mismo tiempo que valoriza y busca su propia coherencia. Sus investigaciones se desarrollaron a partir de la idea de que la identidad es producto de mecanismos mediante los cuales los individuos o los grupos proyectan un sentido sobre su propio ser, creando una relación entre pasado, presente y futuro, y un sentido de continuidad en el flujo cambiante de la existencia. Las identidades se construyen a través de la mediación de las culturas: el repertorio cultural de referencia indica cómo dar sentido en cada momento a la situación y cómo reaccionar frente a ella de forma pertinente.

En la obra colectiva dirigida por Camilleri se definen las estrategias identitarias como:

Los procesos puestos en marcha (de forma consciente o inconsciente) por un actor (individual o colectivo) para llegar a una, o varias, finalidades (definidas explícitamente o situadas a nivel del inconsciente). Son procesos elaborados en función de la situación de interacción (socio-histórica, cultural, psicológica) y de los recursos disponibles (Camillieri, 1990: 24).

Las estrategias identitarias, según este modelo, tienen una doble finalidad: por un lado una finalidad ontológica relacionada con la coherencia entre identidad real e ideal y, por el otro, una finalidad pragmática, que hace referencia al reconocimiento social, acceso a derechos, estatus social, etc.

4 Baumann (2005), sugiere que en lugar de hablar de identidad heredada o adquirida, sería más coherente con la realidad global en la cual vivimos hablar de identificación, de una actividad sin fin, incompleta y abierta, que nos ocupa a todos. 
El esquema de Camilleri para clasificar las diferentes estrategias identitarias prevé dos variables: el objetivo perseguido y la actitud de los sujetos. Los sujetos pueden optar por solo una de las dos culturas o la síntesis cultural. Las actitudes pueden ser de apertura o de repliegue cultural.

TABLA 1

ESQUEMA DE LAS ESTRATEGIAS IDENTITARIAS SEGÚN CAMILLERI

\begin{tabular}{|l|l|l|}
\hline & REFERIRSE A UNA ÚNICA CULTURA & ARTICULAR LAS DIFERENTES CULTURAS \\
\hline Repliegue & $\begin{array}{l}\text { Crispación identitaria, conservadurismo y } \\
\text { repliegue en la cultura de origen. }\end{array}$ & $\begin{array}{l}\text { Sincretismo: asumir elementos de las dos } \\
\text { culturas sin preocuparse por la coherencia. }\end{array}$ \\
\hline Apertura & $\begin{array}{l}\text { Fluidez identitaria y oportunismo: se adopta } \\
\text { de forma instrumental la cultura del país } \\
\text { de recepción. }\end{array}$ & $\begin{array}{l}\text { Integración: se busca una síntesis nueva y } \\
\text { original entre las dos culturas. }\end{array}$ \\
\hline
\end{tabular}

Fuente: Elaboración propia a partir de Camilleri (1990).

En los últimos veinticinco años investigadores e investigadoras franceses como Camilleri, Kastersztein, Lipiansky, Malewska-Peyre, Taboada-Leonetti y Vásquez (1990); Costalascoux, Hily y Vermes (2000); y Manço (2006) han aportado un importante conjunto de herramientas conceptuales sobre las dinámicas identitarias que han influido fuertemente en la perspectiva metodológica y analítica de este estudio. Sin embargo, la finalidad de nuestra investigación es desarrollar su perspectiva teniendo en cuenta no sólo la naturaleza dinámica de la identidad cultural, sino también la porosidad y fluidez de las culturas de referencia. De hecho, el modelo clásico de Camilleri es limitado a la hora de analizar las estrategias identitarias de sujetos como los emigrantes retornados, que vuelven a su país de origen y se encuentran en algunos casos "extranjeros en su tierra». Los emigrantes retornados no tienen que gestionar solamente los conflictos generados del encuentro entre el horizonte cultural canario y venezolano, sino también, entre lo que ellos consideran como horizonte cultural canario y la cultura canaria que encuentran en su regreso, que inevitablemente ha cambiado respecto al período anterior a su emigración. Es más, las fronteras entre horizonte cultural canario y venezolano son, a menudo, fluidas y difícilmente identificables y, en algunos casos, no existen. 


\section{METODOLOGÍA DE LA INVESTIGACIÓN}

En el presente estudio se ha utilizado como modelo de diseño de investigación y de análisis la Teoría Fundamentada (TF) o Grounded Theory, propuesta por Glaser y Strauss (1967). Siguiendo este método se construyen teorías, conceptos, hipótesis y proposiciones que parten directamente de datos y no de supuestos a priorísticos ni de otras investigaciones o de marcos teóricos existentes. El método comparativo constante (MCC), columna vertebral de la Teoría Fundamentada, es más compatible con las finalidades y las características de nuestra investigación, ya que, tal y como subraya Valles (2003: 347-348) no se ocupa de testar una teoría predefinida, sino de generar categorías conceptuales y sus propiedades.

El resultado final de la TF es la delimitación de un conjunto de hipótesis, esto es, un marco teórico que sirva como punto de partida para ulteriores estudios sobre las estrategias identitarias de los emigrantes canarios y de los emigrantes retornados en general. Para obtener este resultado se ha iniciado el análisis de los datos ya desde el comienzo de su recogida. Las primeras hipótesis generadas a partir de los datos iniciales y de la elaboración de los primeros memos $^{5}$ han guiado la búsqueda de nuevos incidentes y el muestreo teórico. El punto de partida de la elaboración teórica ha sido el descubrimiento de la categoría central, es decir, «la categoría o código, que permite vislumbrar la unión o relación entre conceptos teóricos, con el fin de ofrecer una explicación teórica del fenómeno estudiado» (Trinidad, Carrero y Soriano, 2006:36). La categoría que en este estudio respondía a este requisito ha sido el reconocimiento. A partir de la categoría central hemos descubierto los dos procesos sociales básicos: las estrategias pragmáticas y las estrategias ontológicas. Finalmente, a través de la codificación teórica y del método comparativo constante se han identificado algunos factores que condicionan el desarrollo de una estrategia específica.

5 En la teoría fundamentada los memos son el registro sistemático de notas teóricas a partir de los datos recogidos. 


\subsection{Técnicas de recogida de datos}

Las principales técnicas de recogida de datos que se han utilizado han sido la observación participante, las entrevistas a informantes-clave y las entrevistas narrativas.

La observación participante ha estado encaminada a comprender los procesos sociales básicos a través de los cuales los emigrantes retornados canarios redefinen, reconstruyen o reproducen su identidad en un contexto relacional. Se trata de comprender los procesos de identificación, de construcción de una identidad colectiva, de construcción y definición de la «alteridad».

La entrevista narrativa (Atkinson, 1998) ha sido el método de recogida de datos principal de esta investigación, puesto que las narraciones no constituyen un simple intercambio de informaciones producida por un "elaborador de informaciones», sino que son una "producción de sentido» a través de la cual el sujeto presenta y reconstruye su identidad. El lenguaje narrativo permite la estructuración de la experiencia humana. Esta capacidad se hace posible gracias al pensamiento narrativo sistematizado por Bruner (1990): según este autor una de las características fundamentales, y menos valorada, de la experiencia humana, es su condición narrativa. El sujeto como «autor» a través de la narración de su historia se narra a sí mismo, da voz y coherencia narrativa a su identidad y se posiciona en la historia dejando huellas de las estrategias que lleva a cabo para la resolución de conflictos identitarios.

La narración refleja el modo en el cual las personas comprenden e interpretan a los otros seres humanos y sus acciones. Al mismo tiempo, como modalidad de pensamiento intrínsecamente cultural, la narración constituye un medio a través del cual se puede inferir el proceso de construcción del significado (Axia y Moscardino, 2007: 71).

El tipo de entrevista narrativa que se ha diseñado es parecida a la entrevista en profundidad y se encuadra en los tipos life-story: la persona entrevistada cuenta extensivamente sus experiencias a lo largo de todo el arco de la vida o bien puede referirse a un periodo limitado respecto al cual se le pide al entrevistado una narración. Las entrevistas han sido estructuradas haciendo una síntesis de las propuestas de Schutze (1977) y de Flick (1998). A partir de los discursos producidos 
por los actores y las acciones que los actores llevan a cabo, es posible estudiar la identidad. Las identidades tienen una función selectiva, esto significa que los actores eligen, seleccionan diferentes alternativas de acción en base a su identidad, en este sentido:

Es posible imputar un determinado tipo de identidad a un actor social a partir de la observación de ciertas características de su acción (sus preferencias, sus fines, sus estrategias, su estilo, etc.) en un determinado contexto cultural (Giménez, 1996, citado por Rizo, 2004: 113).

Para llevar a cabo la integración final del marco teórico se han recogido sistemáticamente los memos de cada una de las categorías, volviendo a los datos cada vez que necesitábamos validar algún aspecto de la investigación. La comparación continua de los memos y su relación con los códigos teóricos y sus propiedades, han generado un esquema teórico emergente que ha sido modificado y reintegrado hasta que se ha ajustado a los datos recogidos. Finalmente se han comparado los conceptos encontrados con los propuestos por otros investigadores, en particular los que conforman la obra colectiva dirigida por Camilleri (1990). El resultado del estudio es un esquema teórico que, aunque no tenga el estatuto de teoría, sirve como base para futuras investigaciones sobre las dinámicas identitarias de los emigrantes retornados.

\subsection{La muestra}

La población objeto de estudio estaba conformada por emigrantes canarios retornados de Venezuela residentes en la provincia de Santa Cruz de Tenerife ${ }^{6}$. En línea con lo planteado por la teoría fundamentada, en esta investigación se ha utilizado un muestreo teóri$c o$. Se trata de un muestreo que se aleja de la definición cuantitativa de muestreo fundada sobre el concepto de representación. El muestro teórico no acaba hasta que no se llega a la saturación teórica, esto es, cuando los datos nuevos no aportan nueva información y

6 En esta investigación no se han tomado en consideración los emigrantes retornados que han nacido en Venezuela. En este caso, aunque jurídicamente se hable de emigrantes retornados, no hubo una emigración de España sino sólo una inmigración a España. 
empiezan a repetirse. El proceso del muestreo teórico consiste en la codificación, a través del método comparativo constante, de los incidentes hasta que se llegue a la saturación de la teoría, o sea cuando no se ha encontrado ningún tipo de información adicional que permita el desarrollo de nuevas categorías o nuevas propiedades de las categorías.

Además de buscar la saturación hemos tenido en cuenta los siguientes criterios (Valles, 2003): hemos dado prioridad a la expresividad sobre la representatividad según los criterios de heterogeneidad, accesibilidad y recursos disponibles. El resultado final del muestreo teórico ha sido la selección de 22 personas: 11 hombres y 11 mujeres, que migraron a Venezuela entre los 17 y los 33 años de edad, que permanecieron en ese país por un periodo de entre 15 y 45 años, que han regresado a Canarias y que, por último, residen en esta Comunidad Autónoma durante un período de entre 1 a 17 años ${ }^{7}$.

\section{EL RECONOCIMIENTO COMO FINALIDAD DE LAS ESTRATEGIAS}

La codificación abierta ha terminado con el descubrimiento de la categoría central, definida como:

La categoría o código, que permite vislumbrar la unión o relación entre conceptos teóricos, con el fin de ofrecer una explicación teórica del fenómeno estudiado (Trinidad, Carrero y Soriano, 2006: 36).

La categoría central da sentido a todos los datos. Su principal característica es explicar la mayor variabilidad en el patrón de comportamiento social objeto de estudio. La categoría que, en este

7 El trabajo de campo se ha desarrollado entre 2008 a 2011 en la isla de Tenerife. Se han entrevistado con la técnica de entrevista en profundidad a 22 sujetos con una media de seis sesiones por persona. Además se han realizado 63 entrevistas a informantes claves (miembros de asociaciones de emigrantes retornados, emigrantes canarios residentes en Venezuela, familiares y profesionales que trabajan con emigrantes retornados en las islas de La Palma, El Hierro y Venezuela (Caracas, Barquisimeto, Valencia y Chichiriviche). Finalmente se han llevado a cabo 35 sesiones de observación participante. 
estudio, respondía a estos requisitos es el reconocimiento: la necesidad de reconocimiento es el elemento motivacional central que mueve las estrategias identitarias.

Este primer resultado no es una novedad. la centralidad del reconocimiento en las dinámicas identitarias ha sido subrayada tanto en el ámbito filosófico, desde Hegel, hasta las más recientes propuestas de Taylor (1992) y Honneth (2003), como en el de las ciencias sociales:

Sin un «tú» referencial no hay posibilidad de construir una persona, ya que sólo el reconocimiento, esto es, el saberse conocido, aceptado y querido posibilita la exploración de la propia identidad, el respeto a uno mismo y la conciencia de la propia dignidad, la autoestima y autovaloración positiva (Estrada Díaz, 1998: 26).

A lo largo del análisis de las entrevistas narrativas se han identificado cinco dimensiones de reconocimiento, que pueden ser interpretadas como las finalidades básicas de todas las estrategias identitarias:

- Sentimiento de coherencia y continuidad. Lo que Camilleri (1990) denomina necesidad de los sujetos de vivir en una unidad de sentido que permite controlar la realidad. Reconocerse a sí mismo en la continuidad del tiempo es uno de los principales objetivos de las estrategias identitarias: la integración de las diferentes dimensiones de la identidad en una narración coherente. Castells (1998: 28) subraya cómo la identidad «es la fuente de sentido y experiencia para la gente». Gracias a la identidad la persona se reconoce a sí misma como origen de sus pensamientos y acciones, y sus actos se encuadran en una única unidad de sentido.

- Presencia. Se trata de la necesidad de participar en el espacio social que se considera propio, de ser tomado en cuenta. Los emigrantes retornados que han mantenido una fuerte red social en Venezuela, por ejemplo, expresan a menudo la necesidad de participar y estar presente en la vida de sus familiares y amigos que siguen viviendo en América.

- Pertenecer a un grupo, tener un espacio propio en la sociedad o en un grupo. Ser reconocido por los miembros del grupo de referencia como perteneciente al mismo. El reconocimiento, en este sentido, permite a la persona ocupar un lugar en el espacio social. 
- Reconocimiento de la persona como valiosa. Ser valorado positivamente por los miembros de los grupos de referencia y mantener una autoimagen positiva.

- Reconocimiento de los derechos. Ser reconocido significa también que al sujeto se les reconozcan determinados derechos, que tenga acceso a determinados recursos y servicios.

\section{LAS ESTRATEGIAS PRAGMÁTICAS}

A partir de las diferentes dimensiones del reconocimiento hemos identificado dos procesos sociales básicos, dos clases diferentes de estrategia: las estrategias pragmáticas y las estrategias ontológicas.

En determinadas situaciones, cuando están en juego elementos identitarios (valores, símbolos, etc.) que tienen un valor secundario para los emigrantes retornados, estos se concentran en prácticas de auto-presentación encaminadas a lograr sobre todo intereses sociales y beneficios psicológicos: la aprobación, la deseabilidad social o el acceso a determinados recursos. Al contrario, cuando están en juego elementos que consideraban centrales, llevaban a cabo otro tipo de estrategias, menos superficiales y contextuales, dirigidas a defender la integridad de la propia identidad.

Esto es, cuando un sujeto percibe un conflicto entre creencias periféricas (valores, representaciones, actitudes, etc.) activa estrategias pragmáticas y contextuales, al contrario que cuando están en juego creencias y valores centrales, en que se activan estrategias ontológicas.

Las estrategias pragmáticas son formas de auto-presentación en situaciones de interacción, mientras que las estrategias ontológicas son procesos de modificación de la identidad más profunda, entendida como sistema valorativo personal desde el cual la persona se define a sí misma e interpreta el entorno y sus relaciones con ella.

Los términos «estrategia ontológica» y «estrategia pragmática»se inspiran en las propuestas de Camilleri (1990), sin embargo en Camilleri las dos estrategias constituyen dos polos de un continuum: las estrategias pragmáticas tienen una función instrumental y su objetivo es la adaptación al entorno; las estrategias ontológicas tienen como objetivo mantener una coherencia identitaria. En nuestro caso se trata de dos procesos sociales básicos diferentes en los cuales entran 
en juego diversas dimensiones de la identidad: en la pragmática la dimensión más superficial, y en la ontológica la dimensión más profunda. Nuestra propuesta, considerando las dos estrategias como dos procesos diferentes, posibilita la concomitancia de estrategias que maximizan tanto las finalidades pragmáticas cuanto las finalidades ontológicas. En determinados contextos, por ejemplo, en los cuales no están en juego los valores centrales, los sujetos pueden limitarse a adaptarse, mientras que cuando están en juego valores centrales se comprometen con estrategias mucho más complejas e integrativas ${ }^{8}$.

Las estrategias ontológicas son una respuesta a la amenaza a los valores y creencias centrales, lo que se podría considerar el núcleo duro de la identidad. Si bien no es posible definir a priori cuáles son los valores centrales y periféricos de un sujeto, a través del método comparativo constante se han identificado algunos valores que son considerados centrales, en mayor o menor medida, por gran parte de los sujetos estudiados, y que están relacionados especialmente con la idea de familia, del trabajo, de la educación y de las tradiciones. Las estrategias identitarias son una respuesta a la disonancia identitaria que experimenta el emigrante retornado cuando existe un conflicto relacionado con estos valores. Se han identificado al menos cuatro formas diferentes de disonancia identitaria:

- La diferencia entre la identidad cultural real e ideal, que se da, básicamente, cuando la imagen que el emigrante canario tenía de Canarias no corresponde a la realidad del retorno.

- El contraste entre autodefinición e identidad asignada, sobre todo en el caso de emigrantes retornados que se consideran canarios pero son definidos y tratados como extranjeros.

- Los conflictos generados por las diferencias culturales percibidas entre los distintos horizontes culturales: venezolano, canario antes de la migración y canario actual.

- La percepción de la desvalorización de la propia identidad cultural, particularmente la venezolana, por parte del grupo de referencia.

8 La conceptualización de Ralph Turner se asemeja a cuanto planteamos: "por un lado esta la concepción de sí, también denominada identidad; y por el otro, está, la imagen de sí. La primera responde a valores y aspiraciones durables que el individuo percibe como constitutivos de su yo profundo, mientras que la segunda representa la fotografía que registra su apariencia en un determinado instante. La primera es consistente, sin ser inmutable, y la segunda es efímera, variable y plural» (citado por Rizo, 2004: 116). 
El conflicto entre valores centrales genera una disonancia identitaria y puede deberse a la falta de reconocimiento, a la incapacidad de poder actuar coherentemente, o al hecho de que los valores centrales ya no son funcionales en la nueva realidad.

Las estrategias pragmáticas se pueden considerar la dimensión instrumental de la identidad del sujeto. La elección de una estrategia depende, en este sentido, de la previsión del rendimiento de los patrones valorativos en el nuevo contexto. Se activan cuando hay un conflicto entre la autodefinición, la heterodefinición y/o entre los recursos identitarios del emigrante retornado y las características de la situación en la cual está involucrado. Entre las principales finalidades de las estrategias pragmáticas hay que destacar los intereses sociales (el acceso a determinados recursos materiales o simbólicos); y los beneficios psicológicos (valoración positiva, autoestima, reacción a la frustración, al sentimiento de culpa). En este contexto estamos lejos de una visión "ontológica» de la identidad y nos acercamos más a una perspectiva pragmática y funcional. La estrategia identitaria es siempre un posicionamiento situacional y relacional. La auto-presentación depende de la definición de la situación, de la relación con los otros y de las finalidades perseguidas.

Utilizando una metáfora propia de la etnopsiquiatría de Devereux (1972) las estrategias pragmáticas se asemejan a una caja de herramientas: cada "herramienta» es una táctica identitaria que el actor elige, más o menos conscientemente, en función de la situación en la cual está involucrado y de sus objetivos. En este estudio se han ordenado las estrategias pragmáticas elaborando un continuum: conformismo, mimetismo, singularización, diferenciación, resistencia.

Conformismo. Es, probablemente, una de las estrategias más descritas en la literatura sobre identidad y aculturación (Tajfel, 1982; Kastersztein, 1990). No se trata de una asimilación pasiva al grupo de referencia, puesto que conlleva siempre un proceso dialéctico de negociación que pasa por la identificación y uso de los indicadores identitarios que se consideran e interpretan como propios y valorizados por el grupo de referencia. La finalidad es gozar de las ventajas del grupo dominante, en términos de estatus, de autoestima y de recursos y evitar ser identificado con el grupo de emigrantes retornados o de venezolanos o bien, en general, con el grupo que se considera estigmatizado por los canarios. Es importante destacar que no siempre se identifican o se utilizan los indicadores de forma 
pertinente, en estos casos se genera un conflicto entre la autopresentación y la heterodefinición identitaria, el código en vivo más común en estos casos es "yo nací aquí, como tú».

Mimetismo: La finalidad de esta estrategia no es ser identificado como miembro del grupo de referencia, sino más bien ser anónimo. Se trata de minimizar los marcadores identitarios para evitar ser «etiquetado» como miembro de un determinado grupo. Se puede distinguir entre tácticas pasivas de mimetismo y tácticas activas. Entre las pasivas hay que destacar la evitación, por ejemplo, cuando el sujeto en una determinada situación prefiere guardar silencio, no participar o mantenerse al margen para evitar que los demás «descubran» determinados indicadores de su identidad, a través de un determinado tipo de acento, por ejemplo. Por otro lado tenemos el camuflaje, que es una táctica activa de mimetismo donde, por ejemplo, el emigrante retornado se esfuerza en adaptar su comportamiento según las que considera que son las expectativas de cada uno de los grupos ${ }^{9}$.

La Singularización es una estrategia pragmática que rompe la lógica de las identidades opuestas a través de la valoración de la propia singularidad. Los sujetos evitan posibles valoraciones negativas rechazando las categorizaciones culturales y presentándose según «categorías transculturales» como puede ser la profesión: médico/a, abogado/a, agricultor/a. Se pone el foco de atención en otros indicadores que minimizan la atención sobre las diferencias culturales. Otra táctica propia de la estrategia de la singularización es la inconmensurabilidad, que significa que frente a la posible desvalorización la respuesta es presentar las dos «culturas» como incomparables. El código en vivo que expresa esta táctica es: «Venezuela es otro mundo [...] no, mira... es otra vaina».

Diferenciación relativa es la estrategia opuesta al mimetismo. Su finalidad es resaltar la diferencia respecto al grupo con el cual se está interactuando. El emigrante retornado que lleva a cabo esta estrategia resalta sus rasgos venezolanos cuando está con canarios y los rasgos canarios cuando está con venezolanos. Los contextos típicos en las cuales se activa esta estrategia son aquellos donde una situación negativa es claramente relacionada con canarios o con venezolanos.

9 Este fenómeno ha sido denominado principe d'alternance por Guerraoui y Troadec (2000). 
Resistencia: Se trata de un conjunto de tácticas destinadas a crear una identidad que se opone a la identidad mayoritaria. El grupo funciona como círculo de reconocimiento. La estrategia pragmática de la resistencia es activada por grupos en oposición a la identidad dominante o en reacción a la identidad prescrita. Un factor que hay que tener en consideración es que las estrategias identitarias se desarrollan en un sistema de relaciones asimétricas, dónde, a menudo, los emigrantes retornados ocupan la posición más desfavorable. Esto significa que tienen menos poder de negociación a la hora de definir la adscripción a un grupo.

\section{LAS ESTRATEGIAS ONTOLÓGICAS}

Las estrategias ontológicas se han clasificado dependiendo de cómo un sujeto resuelve los posibles conflictos que se generan en un espacio intercultural y cómo gestiona los diferentes elementos propios del horizonte cultural canario y del horizonte cultural venezolano. A través de la codificación teórica se han encontrado cuatro modelos diferentes de gestión de la diversidad: repliegue, asimilación, integración y bricolaje. Los dos primeros se caracterizan por la coherencia simple (Camilleri, 1990): los sujetos resuelven las posibles contradicciones con la supresión de uno de los elementos contradictorios. La tercera y la cuarta se caracterizan por la búsqueda de una coherencia compleja, esto es, los sujetos elaboran estrategias capaces de asumir la contradicción sin suprimir los elementos de oposición.

El desarrollo de una coherencia simple o compleja depende de la interacción de diferentes factores psicosociales entre los cuales hay que destacar: las características de los actores, como el sexo, la edad y sus competencias interculturales; los años transcurridos desde el retorno; el tipo de disonancia identitaria vivida; el proyecto migratorio de retorno y las actitudes del grupo de referencia.

\subsection{La estrategia del repliegue}

El repliegue elimina los conflictos cancelando los nuevos elementos culturales o los elementos ajenos al propio horizonte cultural. Implica una concepción sustancialista de la cultura y una 
percepción de la diversidad como amenaza. En este sentido, esta forma de gestión de la diversidad, ha sido definida por numerosos autores como de oposición (Anolli, 2004). Existen al menos dos variantes de estrategia de repliegue: el eterno migrante y el repliegue venezolano. En el primer caso se trata de emigrantes retornados que se identifican fuertemente con Canarias, pero no con la sociedad canaria actual. Estos emigrantes hubieran deseado encontrar su Isla como la habían dejado décadas antes. Se muestran a menudo críticos con los canarios y con los otros emigrantes retornados, sus discursos tienen un leitmotiv común: la retórica de la autenticidad, sus narraciones se centran en la pérdida de la autenticidad de la sociedad canaria actual que, según ellos, "se echó a perder». El eterno migrante lamenta la pérdida del verdadero significado de las tradiciones:

Hoy en día al canario de aquí le falta respeto a la tierra... anda, pegunta a un agricultor si conoce la mora... si son canarios sólo a la hora de pedir dinero, que no hay gana de trabajar la tierra, que ya no la quiere nadie, que con el turismo ya no hay quién quiera... que por estos vienen los moros aquí, que sino no hay nadie que quiera trabajarla $\left(\mathrm{H} 20-43-2^{10}\right)$.

La persona que activa esta estrategia se identifica profundamente con Canarias, pero con «la Canarias del pasado», cargada de elementos míticos, sus discursos se construyen alrededor de numerosas argumentaciones sobre las causas de la supuesta decadencia de Canarias: el dinero fácil y la falta del "verdadero trabajo». El «dinero fácil» que ha corrompido a los canarios se opone al «dinero sudado», ganado con esfuerzo, constancia y dedicación por el emigrante. La lógica de su argumentación es perfectamente comprensible si recordamos que el trabajo es un elemento central de las representaciones sociales que los emigrantes tienen de sí mismos como canarios: un trabajo de «manos y corazón», "fiel a la tierra». $\mathrm{El}$ «dinero sudado» es una metáfora que trasciende las dimensiones

10 A lo largo del artículo se presentarán algunas frases extraídas de las entrevistas narrativas, especificando si se trataba de un hombre o una mujer, la edad en la cual ha emigrado a Venezuela, los años de permanencia en el extranjero y los años transcurridos desde el retorno. En este caso se trata de un hombre que ha emigrado a Venezuela con 20 años, se ha quedado en este país 43 años y ha vuelto a Canarias desde 2 años. 
económicas, una persona que se gana el dinero con el sudor de la frente se lo ha ganado en un entorno difícil, con honradez, con empeño, y ha comprendido que las cosas tienen valor.

La resolución de los conflictos culturales que implica la puesta en juego de valores considerados centrales, como el trabajo, la familia, la educación de los hijos, la honradez, se resuelve normalmente de forma unilateral. El emigrante retornado se considera a sí mismo como garante de los valores auténticamente canarios en oposición a los valores dominantes en la actual sociedad canaria. Los temas principales de los discursos de las personas que activan esta estrategia son: crítica a los elementos nuevos de la cultura canaria; desconfianza hacia la clase política, en parte culpable de la «corrupción del canario» y desconfianza hacia los extranjeros.

La persona que desarrolla esta estrategia lamenta sentirse doblemente extranjero: no se identifica con Venezuela y no se identifica con la actual sociedad canaria:

Yo, entre Venezuela y Canarias, me he perdido en el Atlántico (H 21-45-2).

Resulta ser una estrategia común en las personas que en Venezuela estaban profundamente identificadas con Canarias y con sus tradiciones y que, una vez retornadas, han experimentado una profunda crisis causada por las contradicciones entre la «Canarias de ataño» idealizada durante décadas en Venezuela y la actual sociedad canaria.

El sentimiento de «doble ausencia» y desarraigo se agrava si el emigrante retornado no cuenta con una red social de apoyo y/o con recursos económicos suficientes. En este caso nos encontramos frente a niveles muy altos de estrés y malestar psicológicos confirmados en las entrevistas a profesionales que trabajan en centros para mayores que acogen a emigrantes retornados.

La segunda forma de repliegue identitario la encontramos entre los emigrantes retornados que están pasando por grandes dificultades de reintegración en la sociedad canaria y el consiguiente empeoramiento de su calidad de vida. Los principales problemas expresados son: el acceso al mercado del trabajo, la falta de reconocimiento de las competencias profesionales, sentimiento de soledad y discriminación. Perciben su situación como más parecida a la de los «inmigrantes latinoamericanos» que a la de los canarios: 
Yo no vine aquí para limpiar, vine porque soy de aquí y porque en Venezuela ya uno no puede vivir... en Venezuela sólo se puede morir. Yo siento que la gente de aquí nos tienen miedo... yo seguro no le quito su trabajo... que yo tengo un trabajo de inmigrante. Limpiando hoteles... dime tú si es un trabajo para una licenciada... esta es mi tierra pero me tratan como una extranjera (M 18-30-2).

Los emigrantes retornados que desarrollan esta estrategia muestran un fuerte resentimiento hacia los canarios. Éstos son considerados los mayores culpables del empeoramiento de su calidad de vida. Hay una simplificación de las atribuciones respecto a las causas del empeoramiento de la calidad de vida. Todos los problemas son debidos a la falta de reconocimiento por parte de los canarios. El repliegue identitario parece ser una forma de resistencia cultural (Hannerz, 1996) frente a la falta de reconocimiento por parte de la sociedad canaria y a las dificultades de normalización de las propias condiciones de vida. El repliegue es, en este caso, una respuesta a la desvalorización de la propia identidad, a la pérdida del estatus social debido al retorno y al rechazo por parte del grupo de referencia.

La estrategia del repliegue identitario se caracteriza por una idealización de los valores venezolanos acompañada por una marcada ostentación de los marcadores identitarios venezolanos. El emigrante retornado rechaza "por principio" cualquier elemento cultural que percibe como canario, activando la que ha sido llamada identité de principe (Manço, 1999).

La estrategia del «repliegue venezolano» suele ser una estrategia colectiva, más que individual, lo que a veces puede limitar la posibilidad de eventuales cambios culturales, en el sentido que el círculo de reconocimiento (Pizzorno, 1989) alimenta y refuerza esta identidad y al mismo tiempo sanciona los posibles cambios.

Un factor añadido de tensión y de estrés para los emigrantes retornados que desarrollan esta estrategia es que sufren lo que se podría definir como una pérdida de continuidad cultural en el interior de su familia. La mayoría de los emigrantes vuelven con sus hijos y éstos viven procesos de interculturalización ${ }^{11}$ que pueden ser bastante

11 Para los individuos y los grupos que pertenecen a dos o más grupos culturales, llamaremos interculturación a los procesos con los cuales, a lo largo de las interacciones que desarrollan, ellos utilizan implícitamente o explícitamente la diferencia cultural que tienden a metabolizar Denoux (1993). 
diferentes respecto a los de los padres. Es común que los emigrantes retornados que desarrollan esta estrategia identitaria a menudo sufran porque los hijos no comparten la misma identificación, los mismos valores, etc. La estrategia pragmática más común entre los retornados que desarrollan esta estrategia es la resistencia.

Finalmente esta estrategia implica lo que Taboada-Leonetti llama le retournement sémantique:

La identidad minoritaria prescrita es aceptada, con todos sus elementos estigmatizantes, pero su significado es objeto de una inversión semántica y se trasforma en positivo [...] aparece a menudo como el primer signo de una toma de conciencia del hecho que la desvalorización de la identidad individual y colectiva es consecuencia de una situación social de discriminación y dominación (Taboada-Leonetti, 1990: 68).

Hay que subrayar que las personas que activan esta estrategia a menudo tienen un proyecto migratorio más parecido a un exilio que a un retorno. Se trata, sobre todo, de personas que han decidido regresar por motivos relacionados con el sentimiento y experiencias de inseguridad vividas en Venezuela y que, en otras circunstancias, no hubieran retornado.

Yo no quería volver a Canarias, tenía que irme de Venezuela (H 21-30-2).

\subsection{La estrategia de asimilación}

Las personas que activan esta estrategia expresan un claro rechazo de los valores propios del horizonte cultural venezolano y se sienten «traicionados» por Venezuela. En la mayoría de los casos se trata de personas que han sido perjudicadas por los cambios económicos y políticos que ha experimentado Venezuela en los últimos diez años y que no encuentran en ese país un contexto adecuado para disfrutar de su vejez.

Las personas que desarrollan esta estrategia aceptan con entusiasmo los nuevos elementos de la cultura canaria y activan un conjunto de tácticas dirigidas a asimilarse al colectivo canario. Entre estas prácticas destacan la utilización de marcadores identitarios canarios (re-apropiarse del acento canario, uso y consumo de 
productos típicos, celebraciones) y la búsqueda de una nueva red social que pueda servir como espejo de sus orígenes canarios. Se preocupan de buscar y frecuentar amigos conocidos antes de la emigración y buscan los lugares que tengan «signos» de su pasado canario: la casa propiedad de los padres, los invernaderos donde habían trabajado, los bares y los clubes que frecuentaban.

El sujeto que desarrolla esta estrategia se preocupa por diferenciarse del resto de los emigrantes retornados y sobre todo de los venezolanos, evita frecuentar personas que puedan «recordar el pasado venezolano», y los contactos con los conocidos que todavía viven en Venezuela. Mantienen una coherencia identitaria eliminando todos los indicios de su pasado migratorio. Esta estrategia resulta problemática cuando el grupo de referencia no reconoce a la persona como parte de su grupo, ya que en este caso se crea una disonancia identitaria que puede ser particularmente dolorosa y difícil de gestionar.

«ER: los canarios somos un pueblo tolerante, abierto y la verdad que yo mismo tengo que decir que cuando regresé me acogieron como un canario más, los problemas se crean porque los que llegan a aquí se pasan, no se dan cuenta que hay que adaptarse y que no puedes ir pidiendo, pidiendo pidiendo sin dar nada, yo cuando llegué... pues que claro aquí las cosas habían cambiado... los años pasan pa'todos, pues yo me adapté y redescubrí cosas que tenía olvidado. Lo que vienen aquí sin gana de adaptarse, creídos, queriendo comerse el mundo... ya sabes cómo son los venezolanos...

EN: ¿Cómo son?

ER: presumidos y esto a los canarios nos fastidia. A mí me cae muy mal esa actitud de «sabelotodo». [EIN: 104: Er].

\subsection{La estrategia del bricolaje}

El bricolaje consiste en crear una yuxtaposición de los elementos que el sujeto considera como los mejores de los diferentes horizontes culturales. El principio unificador es, expresándolo a través de un código en vivo: «tomar lo mejor de cada cultura». El sujeto elimina los elementos que pueden entrar en conflicto y mantiene los elementos que considera mejores. Esta estrategia, ampliamente estudiada por Camilleri (1990), ha sido a menudo llamada «maximización de 
las ventajas» (Anolli, 2004). Se trata de una unión sincrética de los valores, es diferente a la integración porque no busca una síntesis, sino que utiliza los diferentes valores de forma pragmática y oportunista. Sin embargo, al contrario de lo sostenido por Camilleri et al. (1990), el bricolaje no implica falta de coherencia, sino la construcción de lógicas alternativas: frente a las posibles disonancias identitarias hemos codificado diferentes tácticas de gestión de la diversidad que tienen todas como elemento común el bricolaje: la indexicalización cultural (Anolli, 2004), la yuxtaposición de los elementos que el sujeto considera como los mejores de los diferentes horizontes culturales y la negación de las diferencias.

En la indexicalización cultural (Anolli, 2004), que consiste en asumir una identidad «más venezolana» cuando se está en un «entorno venezolano», y una identidad canaria cuando el sujeto se encuentra en un entorno "canario», el principio que da coherencia es la adaptación: frente a las posibles contradicciones entre las diferentes dimensiones de su identidad el sujeto opta por aislar cada una y hacerla visible solamente en determinados contextos, puesto que su finalidad es ser reconocido a través de la adaptación. Se trata de una estrategia parecida al clásico principe de coupure propuesto por el antropólogo Roger Bastide en 1954. Hemos observado que las personas que utilizan esta particular forma de bricolaje dan coherencia a su identidad manteniendo fijos determinados fines y afirmando que estos se pueden lograr a través de diferentes medios, esto es, que el mismo fin, en contextos diferentes, se puede lograr solamente adaptando los medios al contexto. Se trata de la versión ontológica de la estrategia pragmática del mimetismo. El principio de coherencia es la pertinencia, cada valor de la persona es pertinente y eficaz respecto a un determinado contexto cultural.

Además se conservan elementos propios de la sociedad venezolana por motivos oportunistas:

Allí todo es más fácil, te dejan trabajar en paz, no es como aquí con toda esta permisología, tienen que pedir permiso pa'trabajar... allá te montas tu negocio y pa'lante yo prefiero esta libertad, no tener tanta burocracia por medio... pero ya está cambiando la situación allá también (H15-38-3).

La segunda forma de bricolaje es la negación de las diferencias. Puesto que Canarias y Venezuela tienen una larga tradición histórica y cultural común, y la cultura venezolana es bastante visible en 
Canarias, estos sujetos afirman que en realidad no existen muchas diferencias entre una cultura y la otra.

Pero fíjate que aquí es como estar en Venezuela, hay areperas por todos lados, te consigues venezolanos donde sea, y los canarios y los venezolanos somos muy parecidos. Yo me siento venezolano, Venezuela es mi tierra pero mis raíces están aquí, me he criado aquí y nunca me he sentido extranjero en ninguno de los dos sitios... yo me quedo con nuestra forma de ser y con la tranquilidad de Canarias. Yo creo que al final es así que la personas mejoran, tomando lo mejor de una y de la otra cultura $(\mathrm{H}$ 19-35-1).

El sujeto mantiene los elementos que considera más interesantes de cada uno de los horizontes culturales y resuelve las posibles contradicciones negando las diferencias. A menudo esta táctica resulta ser el primer paso hacia nuevas estrategias ontológicas caracterizadas por la búsqueda de la integración entre diferentes elementos.

La estrategia del bricolaje está acompañada comúnmente por la estrategia pragmática del mimetismo: los emigrantes retornados presentan una determinada fachada de su propia identidad solo en los contextos en donde está valorizada.

\subsection{La estrategia de integración}

Se trata de un proceso de síntesis entre los elementos de diferentes horizontes culturales. Es una articulación de la diversidad que permite la creación de una tercera vía, un nuevo horizonte cultural que mantiene los elementos esenciales de los horizontes culturales originarios. La integración se caracteriza por una concepción no sustancial de las culturas, cuyos confines son considerados fluidos y porosos.

La característica común a los sujetos que desarrollan esta estrategia es que han construido hogares transnacionales, y son miembros de familias transnacionales: si bien sus componentes viven separados la mayor parte del tiempo, se mantienen unidos creando un sentimiento de bienestar colectivo y de unidad. Estas familias son importantes para entender las dinámicas identitarias puesto que, 
Las familias en sí mismas son lugares de pertenencia, parte del imaginario unido a partir del cual una familia transnacional mantiene su identidad (Chamberlain y Leydesdorff, 2004: 227).

En este estudio se han identificado dos modalidades de integración cultural que se han denominado intercultural y cosmopolita.

La primera se caracteriza por su «reflexividad metacultural»: una actitud crítica y al mismo tiempo constructiva, hacia las culturas canaria y venezolana, unida a una doble identificación con los dos horizontes culturales. Se trata de personas que han logrado crear una síntesis coherente de su propio repertorio identitario basado en la comprensión de las premisas implícitas de los diferentes marcos culturales de referencia:

Con los años he aprendido a comprender mi forma de ver el mundo, a sentirme cien por ciento canaria y cien por ciento venezolana. A mis hijas creo que le he trasmitido esta síntesis, muy personal, de lo que significa pertenecer a un lugar que va más allá de esta isla (M 22-24-10).

La segunda desarrolla a nivel ontológico lo que la singularización desarrolla a nivel pragmático. Los sujetos no se identifican con ningún horizonte cultural específico, rompen la lógica de la identidad cultural y se identifican con categorías identitarias transculturales o universales, como por ejemplo la identidad profesional o la identificación como «ciudadano del mundo». Uno de los perfiles más comunes de la estrategia cosmopolita es el emigrante que ha inmigrado a diferentes países antes de volver a Canarias. El sujeto que desarrolla esta estrategia no se identifica ni con Canarias ni con Venezuela, elige otras posibles identificaciones que van desde una profesión hasta la singularización:

Yo he vivido en Canarias, Caracas, Madrid y en ningún sitio me he sentido extranjero... ni me he sentido plenamente identificada con ninguno... yo creo que al final somos todos ciudadanos del mundo y en cada sitio hay cosas que merecen la pena y otras que es mejor olvidar (M 19-32-5).

Si me preguntan lo que soy... pues soy músico y padre de familia... no soy ni canario ni venezolano... yo le debo mucho a Canarias y le debo mucho a Venezuela... pero la verdad que no me siento ni de aquí ni de allá... yo comparto intereses y me identifico con mucha gente, pero no por su nacionalidad sino por su modo de ser... (H 22-30-2). 


\section{CONCLUSIONES}

El resultado final de esta investigación ha sido la identificación y categorización de dos procesos sociales básicos relacionados con las dinámicas identitarias: las estrategias pragmáticas y las estrategias ontológicas y las diferentes fases del ciclo identitario de los emigrantes retornados. Finalmente, a través de la codificación teórica y del método comparativo constante se han identificado algunos factores que condicionan la elección de una determinada categoría. Este estudio ha puesto en evidencia que las estrategias identitarias son difícilmente comprensibles a través de las perspectivas tradicionales centradas exclusivamente en la dialéctica entre cultura de origen y cultura del país de acogida.

La redefinición identitaria de los emigrantes retornados es un proceso complejo y dinámico. Se trata de personas que habitan una doble frontera identitaria que les obliga a mediar no solamente entre el horizonte cultural canario y el horizonte cultural venezolano, sino también entre el horizonte cultural canario premigratorio y el actual. El amplio abanico de estrategias utilizadas para dar coherencia a la propia identidad demuestra la capacidad de las personas para adaptarse y el carácter dinámico, cambiante y contextual de la «identidad cultural».

Esta investigación arroja como resultado un esquema teórico basado en el análisis sistemático de testimonios y realidades que puede resultar útil para investigaciones futuras sobre el retorno, así como para ayudar a comprender la compleja dinámica de integración y adaptación recíproca entre los emigrantes retornados y la sociedad canaria, siendo éste un objeto de estudio muy pertinente e interesante en la actual realidad social y cultural de las Islas. A lo largo del proceso de investigación han surgido nuevos interrogantes que, utilizando como base los resultados de esta investigación, pueden ser objeto de investigaciones futuras. En particular creemos que es de gran interés estudiar en profundidad los factores que condicionan las estrategias identitarias, entre los cuales hay que destacar el género, así como profundizar en el papel que juegan las dinámicas familiares transnacionales en los procesos de construcción y reproducción identitaria.

Los emigrantes canarios retornados de Venezuela representan un ejemplo de un fenómeno más general que caracteriza los movimientos migratorios en la era de la globalización: las identidades 
liminales. Se trata de identidades que se desarrollan, como ya hemos dicho, en un espacio intercultural, donde las culturas se cruzan no sólo en el espacio, sino también en el tiempo (en nuestro caso entre el horizonte canario pasado y presente) y donde el mismo espacio del encuentro trasciende los confines nacionales abriéndose a un campo más transnacional. Este estudio muestra cómo, en los espacios interculturales, las personas migrantes desarrollan diferentes estrategias identitarias difícilmente comprensibles a través de las perspectivas tradicionales centradas exclusivamente en las culturas de origen. Más bien al contrario, parafraseando al antropólogo James Clifford (1992: 109), la pregunta fundamental para entender la identidad liminal ya no es «¿de dónde vienes?» sino «¿entre dónde estás?».

\section{BIBLIOGRAFÍA}

Anolli, L. (2004): Psicologia della cultura. Bologna, Il Mulino.

Ascanio, C. (2000): Deconstruyendo olvidos. El proceso migratorio canario desde la perspectiva de género. Recuperado el 15/05/2009, de www.ameri canistas.es/biblo/textos/08/08-083.pdf

Ascanio, C. (2001): Historiografía sobre el proceso migratorio canario: producciones, tendencias y fuentes. Tebeto, Anuario del Archivo Histórico Insular de Fuerteventura, 14, 397-410.

- (2002): Los Canarios en Venezuela. Identidad y diferencia. Tenerife, Centro de Cultura Popular Canaria.

AtкInson, R. (1998): The life story interview. Thousand Oaks, Sage.

Axia, G. y Moscardino U. (2001): Psicologia, cultura e sviluppo umano. Roma, Carocci.

Bauman, Z. (2005): Identidad. Valencia, Publicaciones de la Universitat de València.

Bruner, J. (1990): Act of meaning. Cambridge (Mass.) Londres, Harvard University Press.

CAmilleri, C. (Coord.) (1990): Stratégie identitaire. Paris, PUF.

Carnero, F. y Nuez J. (Coord.) (2011): Emigración y retorno en Canarias. La Laguna, Instituto Universitario de Desarrollo Regional (IUDR).

CASTELls, M. (1998): La era de la información. Economia, sociedad y cultura. El poder de la identidad. Madrid, Alianza.

Chamberlain, M. y LeydesdorfF, S (2004): Transnational families: memories and narratives. Global Networks, 4(3), 227-241.

Clifford, J. (1996): I frutti puri impazziscono. Torino, Bollati Boringhieri. 
Colectivo 78 (1981): Los efectos económicos de un proceso migratorio: La emigración canaria a Venezuela. en Canarias ante el Cambio. Santa Cruz de Tenerife: Universidad de La Laguna y Banco de Bilbao, pp. 129-146.

Costa-Lascoux, J., Hily M. y Vermès, G. (2000): Pluralité des cultures et dynamiques identitaires. Hommage a Carmel Camilleri. Paris, L'Harmattan.

Delgado, G. y Ascanio, C. (1998): Retorno reciente de emigrante canarios. Las Palmas, Cabildo Insular de Gran Canaria.

Deneux, P. (1993): Recherche interculturelle et Psychologie de la différence. Trajectoires et modes d'apprénsions. Paris, L'Harmattan.

Devereux, G. (1972): L'identité ethnique: ses bases logiques et ses dysfonctions. Paris, Flammarion.

ESTRADA, JA. (1998): Identidad y reconocimiento del otro en una sociedad mestiza. México, Universidad Iberoamericána.

FLICK, U. (1998): An introduction to qualitative research. Thousand Oaks, Sage.

Glaser, B. y Strauss, A. (1967): The Discovery of Grounded Theory: Strategies for Qualitative Research. Chicago, Aldine.

Godenau, D. (2011): Retorno y Mercado del trabajo en Canarias. En Carnero, Fernando y Nuez Juan SEbastián. (Coord.) (2011): Emigración y retorno en Canarias. La Laguna, Instituto Universitario de Desarrollo Regional (IUDR).

Guerraoui, Z. y Troadec, B. (2000): Psychologie interculturelle. Paris, Armand. Hannerz, U. (1996): Transnational Connections. Londres, Routledge.

Hernández González, M. (2005): La emigración Canaria a America. Tenerife, Centro de Cultura Popular Canaria.

Honneth, A. (2003): Umverteilung oder Anerkennung?. Fráncfort, Suhrkamp.

Manço, A. (1999): Intégration et identité: Stratégies et positions des jeunes issus de l'immigration. Paris, De Boeck \& Larcier.

- (2006): Processus identitaires et integration. Paris, L'Harmattan.

Martín, J. F. (1992): El proceso migratorio Canarias-América: emigración y retorno. Las implicaciones sociodemográficas, económicas y espaciales a uno y otro lado del Atlántico», en IX Coloquio de Historia Canario-Americana. Las Palmas de Gran Canaria: Cabildo Insular de Gran Canaria pp. $375-478$.

Melucci, A. (1992): Che cosa è «nuovo» nei «Nuovi Movimenti Sociali»?. Sociologia, 26, 271-300.

Mora, J. (2011): La emigración canaria a Venezuela en CARnERo, Fernando y Nuez Juan Sebastián. (Coord.) (2011). Emigración y retorno en Canarias. La Laguna: Instituto Universitario de Desarrollo Regional (IUDR).

- (2011): La emigración canaria a Venezuela en Carnero, F. y Nuez Juan S. (Coord.) (2011). Emigración y retorno en Canarias. La Laguna, Instituto Universitario de Desarrollo Regional (IUDR).

Pizzorno, A. (1989): Algún otro tipo de alteridad: una crítica a las teorías de la elección racional. Sistema, 88, 27-42. 
Rivero, J. L. (1987): Comentarios al problema de la reinserción de los trabajadores emigrantes en el mercado de trabajo del país de origen. El caso de la emigración de canarios a Venezuela». En III Jornadas de Estudios Canarias-America. Santa Cruz de Tenerife: Caja General de Ahorros de Canarias.

Rizo, M. (2004): Prácticas culturales y redefinición de las identidades de los inmigrantes en El Raval (Barcelona): aportaciones desde la comunicación. Tesis Doctoral. Universitat Autònoma de Barcelona.

Schuetze, F. (1977): Die Technik des narrativen Interviews in Interaktionsfeldstudien. Arbeitsberichte und Forschungsmaterialien. Universität Bielefeld.

TAJFEL, H. (1982): Social Identity and Intergroups Relations. Cambridge: Cambridge University Press.

TAYLOR, C. (1992): Multiculturalism and the politics of recognition. Princeton: PUP.

Trinidad, A., Carrero, V. y Soriano, R. M. (2006): Teoría fundamentada "Grounded Theory». La construcción de la teoría a través del análisis interpretacional. Madrid, CIS Cuadernos metodológicos.

VAlles, M. (2003): Técnicas cualitativas de investigación social. Reflexión metodológica y práctica profesional. Madrid, Síntesis. 